(2)

CASE REPORT

\title{
Neuroleptic malignant-like syndrome with a slight elevation of creatine-kinase levels and respiratory failure in a patient with Parkinson's disease
}

This article was published in the following Dove Press journal:

Patient Preference and Adherence

27 February 2014

Number of times this article has been viewed

Li Wei ${ }^{1,2}$

Yinghui Chen ${ }^{1,2}$

'Department of Neurology, Jinshan Hospital, ${ }^{2}$ Department of Neurology, Shanghai Medical College, Fudan University, Shanghai, People's Republic of China
Correspondence: Yinghui Chen Department of Neurology, Jinshan Hospital, Fudan University, I508 Longhang Road, Jinshan, Shanghai 201508, People's

Republic of China

$\mathrm{Tel}+862134189990$

Fax +86 21 57039578

Email yinghuichen@fudan.edu.cn
Abstract: Neuroleptic malignant-like syndrome (NMLS) is a rare but catastrophic complication of drug treatment for Parkinson's disease (PD). Sudden withdrawal and abrupt reduction of antiparkinsonian drugs are major risk factors. Just as its name suggests, the clinical features of NMLS are similar to neuroleptic malignant syndrome, which is a dangerous adverse response to antipsychotic drugs. Both of these conditions can present with hyperthermia, marked muscle rigidity, altered consciousness, autonomic dysfunction, and elevated serum creatine-kinase (CK) levels. However, we describe a special NMLS case with a slight elevation of CK levels and respiratory failure in the full course of her treatment. The patient, a 68-year-old woman with a 4-years history of Parkinson's disease, presented with hyperthermia and severe muscular rigidity. During the course of her treatment, her maximum temperature was extremely high (above $41^{\circ} \mathrm{C}$ ). At the beginning, the diagnosis of NMLS secondary to dopamine decrease was difficult to make, because her initial blood examination revealed that her serum CK levels were mildly elevated and decreased to normal range rapidly. Although antiparkinsonian drugs and supportive treatment were applied, the patient developed an acute respiratory failure in the early course of treatment. This case report highlights that when confronted with Parkinson's patients with high body temperature and muscle rigidity, NMLS should be taken into consideration even if there is no CK elevation. Likewise, the need for supportive care is essential, because its complications are severe, even such as respiratory failure.

Keywords: antiparkinsonian drugs, creatine kinase, parkinsonism-hyperpyrexia syndrome, respiratory failure

\section{Introduction}

Neuroleptic malignant-like syndrome (NMLS), first reported in Japan in 1981, is a rare complication of drug treatment for Parkinson's disease (PD). ${ }^{1}$ This disease is named after neuroleptic malignant syndrome (NMS), a dangerous adverse response to antipsychotic drugs, due to similar clinical manifestations. The syndrome is characterized by such symptoms as hyperthermia, muscle rigidity or tremor, altered consciousness, autonomic instability, and elevated serum creatine-kinase (CK) levels. ${ }^{2}$ The incidence rate has decreased year by year due to doctors' improved knowledge of NMLS. However, we encountered a patient with NMLS in which the serum CK was mildly elevated, and respiratory failure occurred during the early course of treatment.

\section{Case presentation}

A 68-year-old woman diagnosed with PD for 4 years was admitted to our hospital with chief complaints of hyperthermia and severe muscular rigidity. The patient's husband 
reported that she had been on levodopa (600 mg/day), entacapone (300 mg/day), and amantadine (300 mg/day) for almost 2 years. Before her admission, she visited a clinic for progressive impairment of muscular rigidity, where her levodopa was decreased to $125 \mathrm{mg}$ /day. After 2 weeks, the patient's husband found that his wife was so stiff that she could not even get up on her own. When she was admitted to our hospital, the patient was awake, but developed hyperthermia of $39.7^{\circ} \mathrm{C}$, sinus tachycardia of 116 beats/minute, marked muscle rigidity, and aphasia, but her blood pressure was in the normal range $(130 / 80 \mathrm{mmHg})$. Initial laboratory tests did not change markedly. Blood examination showed a white blood cell count of $8.77 \times 10^{9} / \mathrm{L}$ (reference range 3.69-9.16×109/L), CK level was mildly elevated at $144 \mathrm{U} / \mathrm{L}$ (reference range 30-135 U/L). The urine report revealed protein +++ and hematuria+++, suggesting the existence of myoglobinuria. The admission blood culture was negative, so intercurrent infections were ruled out. The immunerelated and malignant diseases had also been ruled out, such as tumor markers ( $\alpha$-fetoprotein/carcinoembryonic antigen/Ca125/Ca199/Ca50/squamous cell carcinoma) and autoimmune antibodies (double-stranded deoxyribonucleic acid/antinuclear antibody/extractable nuclear antigen) were within the normal range. By the next day of hospitalization, the diagnosis of probable NMLS secondary to dopamine reduction was made, and her levodopa was increased to $600 \mathrm{mg} /$ day. The next day, her CK level had decreased to the normal range (Table 1), but the patient deteriorated, with altered levels of blood pressure $(160 / 100 \mathrm{mmHg})$ and a high fever with a peak temperature above $41^{\circ} \mathrm{C}$. On day 3 of her hospital admission, she suddenly developed an acute respiratory failure (45 breaths per minute, oxygen and carbon dioxide partial pressure were $7.55 \mathrm{kPa}$ and $3.79 \mathrm{kPa}$ through nasal catheter, respectively) and disturbance of consciousness (Glasgow Coma Scale score of 4). Her husband agreed to endotracheal intubation and the patient was transferred to the intensive care unit. Cranial computed tomography and magnetic resonance imaging showed atrophic changes

Table I Changes of different enzymes

\begin{tabular}{llll}
\hline $\begin{array}{l}\text { Day of } \\
\text { admission }\end{array}$ & $\begin{array}{l}\text { Creatine kinase } \\
\text { (normal: } \\
\mathbf{3 0 - 1 3 5} \text { U/L) }\end{array}$ & $\begin{array}{l}\text { Creatine } \\
\text { kinase MB } \\
\text { (normal: } \\
\text { 0-16 U/L) }\end{array}$ & $\begin{array}{l}\text { Lactate } \\
\text { dehydrogenase } \\
\text { (normal: } \\
\text { 313-6 I8 U/L) }\end{array}$ \\
\hline 1 & 144 & 12 & 633 \\
2 & 125 & 11 & 307 \\
3 & 97 & 11 & 812 \\
\hline
\end{tabular}

and small focal ischemia. Finally, the patient died of lung infection and heart failure 10 days later.

\section{Discussion}

NMLS is a neurological emergency, with significant morbidity and mortality. Although the incidence rate has decreased year by year due to doctors' improved knowledge of NMLS, studies have shown that mortality in NMLS is between $10 \%$ and $30 \% .^{2}$ Sudden withdrawal and abrupt reduction of antiparkinsonian drugs are major risk factors, especially dopamine. Mounting case reports have demonstrated that many other events may induce NMLS in PD patients, such as dehydration, intercurrent infection, the use of cholinesterase inhibitors, rapid switchover from bromocriptine to pergolide, constipation, and enteral nutrition, because high dietary protein intake can impair the absorption of levodopa. ${ }^{3-7}$ However, there have been reports that a deterioration in parkinsonian symptoms alone may induce NMLS also. ${ }^{8}$ Biochemical studies have shown that low levels of dopamine metabolite (homovanillic acid) in the cerebrospinal fluid may correlate with the possibility of developing NMLS regardless of the dosage of levodopa. ${ }^{9}$ In our patient, the sudden decrease of levodopa may have been the primary cause.

Clinically, NMLS may also be referred to as parkinsonismhyperpyrexia syndrome. It is characterized by such symptoms as hyperthermia, marked rigidity or tremor, and elevated serum CK levels. Actually the serum CK level does not increase dramatically in each case, so this has not been considered a major diagnostic criteria for NMLS. Sometimes, hyperthermia is an initial symptom. A retrospective study in PD patients with NMLS showed that there were no significant relationships among fever, consciousness disturbances, and serum CK levels, which is the same as NMS in psychopaths. ${ }^{8}$ In some exceptional cases of NMS, CK elevation and emergence of muscle rigidity do not appear at the same time. The former is common in the early stage, which may be a useful indicator for early detection of NMS. ${ }^{10}$ In addition, some NMLS cases without fever or altered consciousness have also been reported. ${ }^{8}$ During the course of our patient's treatment, her maximum temperature was extremely high (above $41^{\circ} \mathrm{C}$ ). However, what was different in our patient was that her CK levels never rose obviously, which contrasts with high serum CK levels in NMLS. However, a review of NMS cases associated with clozapine revealed that NMS may present with milder CK elevation. ${ }^{11}$ Since it had been 2 weeks since onset of symptoms when the patient came to our hospital, there might have been a high $\mathrm{CK}$ level at an earlier stage, which decreased to the normal range when she was admitted to our 
hospital. As for the treatment, intravenous fluid infusion, body cooling, and reinstating antiparkinsonian drugs to the same amount before the onset of NMLS immediately, are the three main treatment measures for NMLS. Vitamin $\mathrm{B}_{1}$ can be added as an adjunctive therapy to avoid induction of Wernicke's encephalopathy. Bromocriptine, dantrolene sodium, and apomorphine serve as three important adjuvant drugs that have been reported to be effective. Dantrolene sodium has been reported to reduce marked muscle rigidity for its inhibition of the release of calcium from the sarcoplasmic reticulum. ${ }^{7}$ In this case, respiratory failure occurred in the early course of NMLS due to chest-wall rigidity. Therefore, supportive care in NMS is uncontroversial. Sometimes, admission to the intensive care unit is necessary due to the nature of the required monitoring and supportive treatment.

To prevent NMLS, there are two aspects requiring attention. Firstly, in order to improve patients' poor compliance with taking medicine, health education must be enhanced. Doctors should remind their PD patients not to withdraw or decrease their antiparkinsonian drugs without authorization. Also, if there is a sudden higher temperature in relation to a recent reduction in medication dose, patients should contact their doctor immediately, for timely treatment can reduce the mortality significantly. Secondly, the doctor must be cognizant of the narrow safety margin for antiparkinsonian drugs, for even a small reduction in these can induce NMLS, especially during acute illness and hospital admissions. When doctors are in these situations, awareness of this disease is most important, because prompt treatment with oral or nasogastric levodopa or subcutaneous apomorphine is life-saving.

\section{Conclusion}

It has been well accepted that elevation of CK levels is one of the symptoms of NMLS in PD patients, but there are exceptional cases, like the case we describe here. For this reason, physicians should take into consideration the possibility of
NMLS when confronted with Parkinson's patients with high body temperature, even if there is milder CK elevation. In addition, supportive care in NMS is necessary to prevent complications.

\section{Disclosure}

The authors report no conflicts of interest in this work.

\section{References}

1. Toru M, Matsuda O, Makiguchi K, Sugano K. Neuroleptic malignant syndrome-like state following a withdrawal of antiparkinsonian drugs. J Nerv Ment Dis. 1981;169:324-327.

2. Ghosh R, Liddle BJ. Emergency presentations of Parkinson's disease: early recognition and treatment are crucial for optimum outcome. Postgrad Med J. 2011;87:125-131.

3. Grace JB, Thompson P. Neuroleptic malignant like syndrome in two patients on holinesterase inhibitors. Int J Geriatr Psychiatry. 2006;21:192-194.

4. Reimer J, Kuhlmann A, Müller T. Neuroleptic malignant-like syndrome after rapid switch from bromocriptine to pergolide. Parkinsonism Relat Disord. 2002;9:115-116.

5. Bonnici A, Ruiner CE, St-Laurent L, Hornstein D. An interaction between levodopa and enteral nutrition resulting in neuroleptic malignant-like syndrome and prolonged ICU stay. Ann Pharmacother. 2010;44:1504-1507.

6. Ogawa E, Sakakibara R, Kishi M, Tateno F. Constipation triggered the malignant syndrome in Parkinson's disease. Neurol Sci. 2012;33: $347-350$.

7. Ikebe S, Harada T, Hashimoto T, et al. Prevention and treatment of malignant syndrome in Parkinson's disease: a consensus statement of the malignant syndrome research group. Parkinsonism Relat Disord. 2003;9:47-49.

8. Hashimoto T, Tokuda T, Hanyu N, Tabata K, Yanagisawa N. Withdrawal of levodopa and other risk factors for malignant syndrome in Parkinson's disease. Parkinsonism Relat Disord. 2003;9:25-30.

9. Ueda M, Hamamoto M, Nagayama H, Okubo S, Amemiya S, KatayamaY. Biochemical alterations during medication withdrawal in Parkinson's disease with and without neuroleptic malignant-like syndrome. J Neurol Neurosurg Psychiatry. 2001;71:111-113.

10. Nisijima K. Elevated creatine kinase does not necessarily correspond temporally with onset of muscle rigidity in neuroleptic malignant syndrome: a report of two cases. Neuropsychiatr Dis Treat. 2012;8: 615-618.

11. Sachdev P, Kruk J, Kneebone M, Kissane D. Clozapine-induced neuroleptic malignant syndrome: review and report of new cases. J Clin Psychopharmacol. 1995;15:365-371.
Patient Preference and Adherence

\section{Publish your work in this journal}

Patient Preference and Adherence is an international, peer-reviewed, open access journal focusing on the growing importance of patient preference and adherence throughout the therapeutic continuum. Patient satisfaction, acceptability, quality of life, compliance, persistence and their role in developing new therapeutic modalities and compounds to

\section{Dovepress}

optimize clinical outcomes for existing disease states are major areas of interest. This journal has been accepted for indexing on PubMed Central. The manuscript management system is completely online and includes a very quick and fair peer-review system. Visit http://www.dovepress.com/ testimonials.php to read real quotes from published authors. 